\title{
植物与病原微生物互作分子基础的研究进展
}

程㬢，田彩娟，李爱宁，邱金龙

中国科学院微生物研究所植物基因组学国家重点实验室, 北京 100101

摘要: 植物在与病原微生物共同进化过程中形成了复杂的免疫防卫体系。植物的先天免疫系统可大致分为两个 层面。第一个层面的免疫基于细胞表面的模式识别受体对病原物相关分子模式的识别, 该免疫过程被称为病原 物相关分子模式触发的免疫(PAMP-triggered immunity, PTI), 能帮助植物抵抗大部分病原微生物; 第二个层面 的免疫起始于细胞内部, 主要依靠抗病基因编码的蛋白产物直接或间接识别病原微生物分泌的效应子并且激 发防卫反应, 来抵抗那些能够利用效应子抑制第一层面免疫的病原微生物, 这一过程被称为效应子触发的免疫 (Effector-triggered immunity, ETI)。这两个层面的免疫都是基于植物对 “自我” 及“非我”的识别，依靠 MAPK 级联等信号网络, 将识别结果传递到细胞核内, 调控相应基因的表达, 做出适当的免疫应答。本文着重阐述了 植物与病原微生物互作过程中不同层面的免疫反应所发生主要事件的分子基础及研究进展。

关键词: 植物; 病原微生物; 先天免疫; 抗病基因; 信号转导

\section{Advances on molecular mechanisms of plant-pathogen interactions}

\author{
CHENG Xi, TIAN Cai-Juan, LI Ai-Ning, QIU Jin-Long
}

State Key Laboratory of Plant Genomics, Institute of Microbiology, Chinese Academy of Science, Beijing 100101, China

\begin{abstract}
Plants have established a complicated immune defense system during co-evolution with pathogens. The innate immune system of plants can be generally divided into two levels. One, named PAMP-triggered immunity (PTI), is based on the recognition of pathogen-associated molecular patterns by pattern-recognition receptors, which confers resistance to most pathogenic microbes. The other begins in cytoplasm and mainly relies on recognition of microbial effectors by plant resistance proteins in direct or indirect ways, which then initiates potent defense responses. This process, termed effector-triggered immunity (ETI), is necessary for defense against pathogens that can secret effectors to suppress the first level of immunity. Activation of these two layers of immunity in plant is based on distinguishing and recognition of "self" and "non-self" signals. Recognition of "non-self" signals can activate signal cascades, such as MAPK cascades, which will then induce defense gene expression and corresponding defense responses. In this review, we focused on underlying molecular mechanisms of plant-pathogen interactions and the latest advances of the PTI and ETI signaling network.
\end{abstract}

Keywords: plant; pathogen; innate immunity; resistance gene; signaling pathway

收稿日期: 2011-05-12; 修回日期: 2011-07-27

基金项目: 中国科学院知识创新工程重要方向项目(编号: KSCX2-EW-J-6)资助

作者简介: 程犧, 博士研究生, 专业方向：植物和病原微生物互作。Tel: 010-64807403: E-mail: chengx02@163.com

通讯作者: 邱金龙, 研究员, 中科院“百人计划” 入选者, 专业方向：植物与病原微生物分子互作。E-mail: qiujl@im.ac.cn 网络出版时间: 2012-1-5 10:17:25

URL: http://www.cnki.net/kcms/detail/11.1913.R.20120105.1017.001.html 
植物在与病原微生物共同进化过程中逐渐形成 了先天免疫系统, 该系统包含复杂的识别机制, 使 植物通过感知“非我” 分子来启动防卫反应抵抗外 来入侵者。植物先天免疫系统包含两个层面：第一 个层面是基于细胞表面的模式识别受体(Patternrecognition receptors, PRRs)对病原物相关分子模式 (Pathogen-associated molecular patterns, PAMPs)的识 别, 在此过程中植物对许多病原微生物中普遍存在 的分子作出识别及应答, 该免疫过程被称为病原物 相关分子模式触发的免疫(PAMP-triggered immunity,

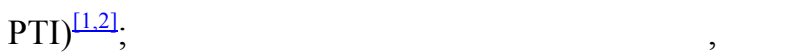
依靠抗病基因(Resistance gene, R gene)编码的蛋白 产物起作用。这些蛋白大部分是NB-LRR (Nucleotide binding-leucine rich repeat)蛋白, 不同的NB-LRR蛋 白可以直接或间接识别不同来源的病原物效应子, 并且激发相似的防卫反应, 这一过程被称为效应子 触发的免疫(Effector-triggered immunity, ETI) ${ }^{[3]}$ 。这 两个层面免疫应答的分子机制各不相同, 又相互联 系, 充分体现了植物与病原微生物互作的复杂性。

植物与病原微生物之间相互作用并协同进化的 过程可分为不同阶段：在第一阶段, 病原微生物通 过各种策略进攻植物, 植物进化出 PRRs识别其中绝 大多数病原微生物的PAMPs，从而触发PTI; 第二阶 段, 少数病原微生物可以通过分泌效应子来抑制 PTI, 再次实现对植物的侵染, 此时植物对病原微生 物是感病的; 第三阶段, 植物进化出能够特异性识 别相应效应子的R蛋白, 触发ETI, 使植物体再次表 现出更强的抗性, 通常在侵染位点产生过敏性细胞 死亡反应(Hypersensitive cell death response, HR), 从而阻止病原微生物的进一步侵染。之后, 在自然 选择的作用下, 病原微生物可能通过不同进化策略 来避开ETI, 进而植物又共进化出新的 R蛋白来再次 触发ETI。从长期的角度看, 植物与病原微生物之间 的互作呈现Z字形的“拉锯战” 局面 ${ }^{[1]}$ 。

对植物与病原微生物之间相互作用的分子机制 深入透彻的解析, 将对植物抗病基因的研究及农作 物品种改良具有指导性的意义。本文以植物对病原 微生物的识别及信号转导不同阶段所发生的主要事 件为线索, 对目前已知的分子机制进行综述。此外, 植物识别病原微生物、在侵染部位迅速启动 PTI 和 ETI 抗病反应的同时, 还产生系统信号分子。该信号
分子通过一定的方式能传递到植物未被侵染的部分, 使之获得对外界病原微生物长期、广谱的抗性。这 种现象被称为系统获得性抗性 (Systemic acquired resistance, SAR)。系统获得性抗性是植物与病原微 生物互作的一个重要过程, 但本文没有具体涉及, 请读者参见国内、外相关内容的综述。

\section{PAMPs 及其识别}

PAMPs是病原微生物表面存在的一些保守分 子。因为这些分子不是病原微生物所特有的, 而是广 泛存在于微生物中 ${ }^{[4]}$, 它们也被称为微生物相关分子 模式(Microbe-associated molecular pattern, MAMPs)。 PAMPs对维持微生物的基本生物学特征十分重要, 并且很少发生变异。真菌PAMPs包括麦角甾醇、多 聚半乳糖醛酸内切酶、木聚糖酶, 以及细胞壁衍生 物葡聚糖和几丁质等; 卵菌PAMPs有 $\beta$-葡聚糖及转 谷氨酰胺酶等; 细菌PAMPs包括脂多糖、冷休克蛋 白、延伸因子 Tu及鞭毛蛋白等 ${ }^{[5,6]}$ 。PAMPs仅由微生 物产生, 不存在于宿主中, 因此宿主可以将它们识 别为“非我”成分, 并产生相应抗性 ${ }^{[7]}$ 。植物依靠其细 胞表面的PRRs来感知PAMPs, 其中研究最为清楚的 PAMPs及其相应PRR 是细菌鞭毛蛋白及拟南芥的 FLS2。

鞭毛蛋白是构成细菌鞭毛的粒状蛋白。Felix等 ${ }^{[8]}$ 通过对铜绿假单胞菌(Pseudomonas aeruginosa)的序 列分析, 发现其鞭毛蛋白 $\mathrm{N}$ 端含有 22 个氨基酸的一 个肽段(flg22)具有激发子活性, 该区域在革兰氏阴 性菌中是高度保守的。在模式植物拟南芥中, 负责 识别鞭毛蛋白的PRR是富含亮氨酸重复序列的类受 体蛋白激酶(Leucine-rich repeat receptor-like kinase, LRR-RLK) FLS2(Flagellin-sensing 2) ${ }^{[9]}$ 。LRR-RLK是 一类单跨膜蛋白, 通常由LRR膜外功能区, 跨膜区 及胞内丝氨酸/苏氨酸蛋白激酶区域组成。FLS2 特 异性识别flg22 并与其相结合。虽然FLS2 蛋白LRR 区域第 9 15 个氨基酸对 flg 22 的结合及应答是必需 的, 但flg22 精确的结合位点还不明确 ${ }^{[10]}$ 。在所有已 知基因组信息的高等植物中都发现了 FLS2 的同源 物。现已证明烟草、番茄及水稻中FLS2 同源蛋白对 鞭毛蛋白识别均有作用, 说明植物通过FLS2 同源物 来识别鞭毛蛋白, 这种方式在进化上是古老而保守 的 ${ }^{[11 ~ 13]}$ 。在拟南芥中, FLS2 基因的突变使植物对致 
病菌Pseudomonas syringae pv. tomato DC3000 (Pst DC3000)更加感病; 同时, 在fls2 突变体上接种非致 病菌Pseudomonas syringae pv. phaseolicola (Pph, 种大豆病原菌)后发现, 与野生型相比, $P p h$ 在突变体 上致病性显著提高 ${ }^{[14]}$ 。沉默烟草NbFLS2 基因也能导 致烟草对一些致病菌及非致病菌的感病性增强 ${ }^{[12]}$ 。 此外, 一些flg22 发生突变的病原菌能够成功避开植 物的识别, 这进一步证明鞭毛蛋白识别在植物-微生 物互作中的重要性和保守性。

用不含鞭毛蛋白但存在其他PAMPs的细菌提取 物处理fls2 突变体, 再对其接种Pst DC3000, 发现细 菌生长依然受到抑制 ${ }^{[15]}$, 表明植物中还存在其他受 体来识别除鞭毛蛋白以外的PAMPs。另一个能被拟 南芥及十字花科其他成员识别为 PAMP的成分, 是 在细菌中大量存在的蛋白一延伸因子 $\mathrm{Tu}$ (Elongation factor Tu, EF-Tu)。EF-Tu具有一个高度保守的 肽段elf18, 它含有 18 个氨基酸并在其N端乙酰化。 elf18 所触发的免疫应答与全长 EF-Tu一致。植物线 粒体或质体EF-Tu没有PAMP活性, 说明植物对细菌 EF-Tu的识别具有特异性。虽然大部分植物对鞭毛蛋 白有应答，但仅十字花科植物对EF-Tu具有敏感性。 拟南芥中负责识别EF-Tu的PRR是 EFR (LRR-RLK EF-Tu receptor), 它与FLS2 同属于LRRXII亚家族 [16]。efr 突变体对根癌农杆菌 (Agrobacterium tumefaciens)及弱致病力的Pst DC3000 感病性增强, 证 明EFR对抵抗细菌具有十分重要的作用。有趣的是, 虽然烟草并不应答EF-Tu，但在烟草中表达拟南芥 $A t E F R$ 基因后, 就能够导致elf18 与其结合并产生相 应免疫应答，表明PRRs的下游信号转导成分在不同 植物中可能是保守的 ${ }^{[17]}$ 。EF-Tu具有典型PAMP的所 有特征：表达量高; 在多种细菌中具有高度保守序 列; 对微生物的存活至关重要。虽然EF-Tu在细菌中 的主要作用是参与mRNA翻译, 但它却能被宿主质 膜上的EFR识别, 这便出现一个问题: 植物怎么能感 知到细菌内部的EF-Tu ? 一种假设是, EF-Tu在细菌 中大量存在, 而EF-Tu受体的敏感性是皮克级别, 当 细菌在植物上繁殖时, 有些细胞裂解会释放出足够 量的EF-Tu，从而被EF-Tu受体识别。但是越来越多 的证据表明，虽然EF-Tu没有典型的信号及运输序 列, 但它也能够在细胞表面定位 ${ }^{[18,19]}$ 。因此, EF-Tu 的分泌过程还有待进一步的研究。
FLS2 和EFR是拟南芥中仅知的两个PRRs。在其 它植物中, 还发现另外一些PAMPs识别受体。最近, 水稻白叶枯病菌 (Xanthomonas oryzae pv. Oryzae)中 一个包含 194 个氨基酸的蛋白 $\mathrm{Ax} 21$ 被鉴定为PAMP, 其 $N$ 末端具 17 个氨基酸的硫酸化肽段 $a x Y^{\mathrm{S}} 22$ 在所有 黄单胞菌属细菌中都是完全保守的。 $\mathrm{axY}^{\mathrm{S}} 22$ 对 $\mathrm{Ax} 21$ 生物活性是必需的, 而在水稻中发现XA21 蛋白能 够结合并识别 $\mathrm{ax} \mathrm{Y}^{\mathrm{S}} 22$ 。XA21 蛋白是一个类受体激酶, 也属于LRRXII亚家族。最初在水稻中克隆到Xa21 基因时, 认为它是一个 R 基因, 能够激活 ETI; 而在 近期研究中发现XA21 是直接与 $A x 21$ 相结合的, 它 是 $A \times 21$ 的模式识别受体 ${ }^{[20]}$ 。

乙烯诱导的木聚糖酶 (Ethylene-inducing xylanase, EIX)是一种真菌PAMP。EIX在番茄中的识别 受体是由LeEIX1/2 基因编码的LeEIX1 和LeEIX2, 这两个蛋白都是类受体蛋白(Receptor-like proteins, $R L P s)^{[21]}$ 。RLPs也具有胞外亮氨酸重复序列及跨膜 区域，但在胞质内缺少蛋白激酶区域，故有可能与 激酶一起作用于下游的信号转导 [22]。在烟草中瞬时 表达LeEIX1 和LeEIX2, 发现它们分别能与EIX相结 合, 但只有LeEIX2 能够转导信号。利用反向遗传学 方法在拟南芥中还发现一些和先天免疫相关的RLPs, 如几丁质诱导的RLP52 基因, 其突变能引起拟南芥 对致病及非致病白粉菌的感病性增强 ${ }^{[23]}$ 。这些和抗 病相关的RLPs是否能作为PAMPs识别受体来起作用 则需更进一步的研究。

在豆科植物中, 一个可溶性 $\beta$-葡聚糖结合蛋白 (Soluble b-glucan-binding protein, GBP)能与大豆疫 霉菌(Phytophtora sojae)细胞壁上 1,6-1,3- $\beta$-葡聚糖 特异性结合。与P. sojae接触时, 具有 $1,3-\beta$-葡聚糖酶 活性的GBP可能与一个未知的跨膜蛋白相互作用来 完成其识别功能 ${ }^{[24]}$ 。几丁质是大多数高等真菌细胞 壁主要组成成分，其结构为 $\beta$-聚 $\mathrm{N}$-乙酰葡糖胺，源 于几丁质的 N-乙酰几丁寡糖是许多植物的PAMP。水 稻几丁质结合蛋白 $\mathrm{CEBiP}$ (Chitin elicitor binding protein)是带有两个胞外LysM基序的跨膜蛋白，与 RLPs相似, CEBiP也缺少胞内蛋白激酶区域 ${ }^{[25]}$ 。将 $C E B i P$ 基因沉默后，发现水稻对几丁质的结合及应 答减少, 证明CEBiP蛋白是几丁质的PRR。在水稻中 鉴定出CEBiP之后不久, 通过反向遗传学手段在拟南 芥中也鉴定出带有 3 个胞外LysM基序的受体激酶 
CERK1(LysM-containing chitin elicitor receptor kinase $1)$, 并证明它对几丁质应答是必需的 ${ }^{[26,27]}$ 。拟南芥 cerk1 突变体表现出对致病真菌Erysiphe cichoracearum及Alternaria brassicicola的感病性增强。然而, cerk1 突变体对致病细菌Pst DC3000 也更加感病 ${ }^{[28]}$, 表明CERK1 不仅作用于几丁质识别, 也参与了细菌 PAMPs的识别,但其识别机制还需要更深入的研究。

\section{PTI 信号通路}

植物 PRRs 对病原微生物 PAMPs 的识别, 仅仅 是它们做出免疫应答的开始。当在细胞膜上确定有 病原菌来袭后, PRRs 还需要和其他质膜蛋白一起作 用, 将这个信号通过相应的信号转导途径传递到下 游, 从而使植物做出进一步免疫应答。

BAK1(BRI1 associated receptor kinase 1)是一个 在多种信号转导途径中普遍存在的接头蛋白, 它也 参与了 PTI应答。BAK1 又被称为SERK3，属于SERK 家族, 该家族由 5 个密切相关的LRR-RLKs组成 ${ }^{[29]}$ 。 BAK1 有 5 个亮氨酸重复序列, 最初它是作为油菜 素内酯受体BRI1 的共受体而被发现 ${ }^{[30]}$ 。当用flg22 处理时, FLS2 和BAK1 能在 $2 \mathrm{~min}$ 之内形成寡聚体; 拟南芥bak1 突变体对flg22、elf18 及其他PAMPs的敏 感性降低, 但并不影响flg22 与FLS2 的结合; 烟草中 $B A K 1$ 的功能缺失也能减少对细菌CSP22 的应答。这 些实验都证明BAK1 参与FLS2 及EFR介导的信号转 导途径, 并起到正调控作用。BAK1 突变对elf18 应答 的影响较弱, 推测EFR可能优先与SERK家族的其他 成员相作用 ${ }^{[31,32]}$ 。

FLS2 和BAK1 都是跨膜蛋白激酶, 它们的底物 是什么? FLS2-BAK1 受体复合体又是怎样将PAMP 信号从细胞膜上传递到细胞内的 ? BIK1 的发现回 答了这些问题。BIK1(Botrytis-induced kinase 1)是e RLK/Pelle/IRAK蛋白激酶家族的一员，该家族的成 员广泛参与了植物、昆虫及人类的先天免疫应答 ${ }^{[33]}$ 。 在植物中, 最初将BIK1 鉴定为一个抗腐生型真菌成 分 ${ }^{[34]}$; 之后发现, 用 flg22、elf18 及几丁质处理的 bik1 突变体，其防卫反应严重受损，表明BIK1 在多 种PRRs所产生不同信号的整合方面起到了重要作 用。用 flg 22 处理植物时, BIK1 的 $\mathrm{Thr}^{237}$ 能够以 FLS2/BAK1 依赖的方式快速磷酸化。体外体内实验 都证明BIK1 能分别与FLS2 和BAK1 相结合, BIK1
是BAK 1 的底物, 然而FLS2 和BAK 1 也是BIK 1 的底 物。据此, $\mathrm{Lu}$ 等提出一个相互作用模式 : 当不存在 鞭毛蛋白(flg22)时，BIK1 以无活性的形式分别与 FLS2 和BAK1 相结合; 鞭毛蛋白与FLS2 相结合后, flg22 诱导FLS2 和BAK 1 结合并磷酸化, 形成受体复 合体; 激活的BAK1 磷酸化BIK1, 之后BIK1 又转而 磷酸化FLS2-BAK1 复合体; 完全激活的FLS2-BAK1 可能进一步磷酸化BIK1 及其他底物; 之后，激活的 BIK1 从FLS2-BAK1 复合体上释放下来, 去激活细 胞内下游信号转导成分 ${ }^{[35,36]}$ 。在原生质体中, BIK1 也具有与EFR及 CERK1 相互作用的能力; 除 flg22 外, EF-Tu也能诱导BIK1 磷酸化。以上实验证据表明, BIK1 参与多种PAMPs受体复合体介导的PTI信号转 导。

丝裂原活化蛋白激酶(Mitogen-activated protein kinase, MAPK)级联是真核生物信号传递网络中的 重要途径之一, 它通常由 3 个蛋白激酶MAP3KMAP2K-MAPK组成级联, 通过依次磷酸化将上游 信号传递至下游应答分子。MAPK级联在动、植物 先天免疫应答不同生物或非生物压力反应的信号转 导中都发挥着重要作用, 其介导的信号转导是植物 对病原微生物产生抗性的关键步骤。拟南芥基因组 约有 110 个基因编码MAPK级联中的成分：20个 MAPKs, 10 个MAPKKs及 80 多个MAPKKKs ${ }^{[37]}$, 其 中MPK3、MPK4 及MPK6 已被证明能为应答PAMPs 诱导而被快速激活 [38]。一系列分子遗传学研究表明, 在植物免疫应答过程中MPK3、MPK4 及MPK6 分别 参与了两条不同的MAPK级联。

在拟南芥中, MPK4 与其上游的MKK1/MKK2 及MEKK1 形成一条防卫反应的信号通路, 这条通 路上的功能缺失突变均能致使植物矮化并导致防卫 反应的组成性激活 ${ }^{[39,40]}$ 。MKS1 被鉴定为MPK4 的 底物，而在酵母双杂交体系中WRKY33、WRKY25 又与MKS1 相互作用 ${ }^{[41]}$; 在体外也检测到WRKY33 与MKS1 的相互作用 ${ }^{[42]}$ 。虽然最初没有观察到MPK4 与WRKY33 的相互作用, 但近期研究证明, WRKY33 以MKS1 依赖的方式与MPK4 相互作用, 核提取物 的免疫共沉淀表明存在MKS1-MPK4-WRKY33 三复 合体 ${ }^{[43]}$ 。当病原物侵染时, 被激活的MPK4 能够磷 酸化MKS1, 致使WRKY33 从该复合体上释放下来, 游离的WRKY33 诱导其靶基因 $P A D 3$ 的表达，从而 
完成相关的防卫反应 ${ }^{[43]}$ 。

当用flg22 处理拟南芥时, MPK3/MPK6 也能被 激活, 但它们的激活不依赖于MEKK $1^{[44]}$ 。组成型激 活的MKK4/ MKK5 能够激活MPK3/MPK6, 从而导 致FRK1(flg22-induced receptor-like kinase 1)、WRKY22 及 $W R K Y 29$ 的转录激活 ${ }^{[45]}$ 。因此, 当PAMPs被植物 识别后, 也激活了一条与 MEKK1-MKK1/MKK2MPK4 平行的级联, 这条级联包括MKK4/MKK5 及 MPK3/MPK6, 形成一条正调控植物防卫反应的信 号通路 ${ }^{[46]}$ 。MKK4 与MKK5 及MPK3 与MPK6 存在 功能冗余, 但 $m k k 4 / m k k 5$ 和 $m p k 3 / m p k 6$ 双突变体均为 致死突变, 这增加了研究的难度。在灰霉菌Botrytis cinerea 侵染拟南芥的过程中, MPK3 对植保素的合 成是必需的, 而 $m p k 3$ 突变体表现出对病原菌的感病 性增强 [47]。最新研究证明, WRKY33 作为MPK3/6 的底物, 在MPK3/6 下游作用于植保素合成基因的 调控。用B. cinerea接种拟南芥时, 可诱导MPK $3 / 6$ 信 号通路的激活, 进而激活WRKY33; 在WRKY33 调 控下, 激活 $P A D 3$ 基因的转录, 大量合成植保素, 从而 做出防卫应答 ${ }^{[48]}$ 。这也暗示MPK4 级联与MPK3/6 级 联可能存在交叉互作。VIP1 (VirE2- interacting protein 1)被鉴定为与农杆菌毒性蛋白 VirE2 相互作用的宿 主蛋白, 农杆菌劫持VIP1 并在其协助下将T-DNA运 输至宿主细胞核内 ${ }^{[49,50]}$ 。然而, 酵母双杂交篮选将 VIP1 鉴定为MPK3 的一个底物, 通过MPK3 能对 VIP1 进行PAMP诱导的磷酸化, 这种磷酸化作用使 VIP1 进入细胞核，核内的VIP1 可能通过调控防卫基 因的表达来抵抗农杆菌的侵染 ${ }^{[50]}$ 。在植物中, PAMPs 能够诱导 1000 多种基因的转录激活, 因此, 还需要 更多研究来确定VIP1 在这种转录调控中的作用范 围。同时, 也可能存在其它转录因子参与PTI过程。

目前, 虽然没有证据表明 PRRs 及其共受体能 与 MAPK 级联上的成分直接相互作用, 但在 PRRs 下 游可能存在其它膜偶联蛋白介导 MAPK 级联的激活。

\section{3 病原微生物对 PTI 的抑制}

植物PTI成功抵挡了大部分病原微生物, 然而 少数病原微生物则进化出相应的策略, 它们通过效 应子抑制PTI, 从而成功展开进一步入侵。那么, 这 些效应子怎样来实现抑制PTI的作用? 有一些效应 子可能在真菌及卵菌侵染植物细胞并形成吸器外基
质的过程中起结构作用 ${ }^{[51]}$; 另一些效应子可能促进 病原物扩散或植物细胞养分渗漏 ${ }^{[52]}$; 而许多效应子 则对 PTI过程中一个或多个成分具有直接或间接的 抑制作用。

许多植物致病细菌, 如P. syringae, 都具有III型 分泌系统(Type III secretion system, TTSS)。细菌效应 子蛋白通过TTSS进入植物细胞, 每种致病菌能利用 TTSS向宿主中递送 15 30 个效应子。细菌效应子经 常通过模仿或抑制真核生物的细胞功能来帮助病原 菌侵染宿主。在植物病原细菌中, TTSS是由 $h r p$ 基因 编码的 ${ }^{[53]}$, 细菌 $h r p$ 突变体能够使易感植物产生防 卫反应，而正常致病菌能抑制这些反应，这首次证 明效应子介导PTI抑制 ${ }^{[54]}$ 。DC3000 效应子AvrPto 及 AvrPtoB在拟南芥及烟草中的表达能够抑制防卫反 应并促进细菌繁殖; AvrPto 及AvrPtoB抑制PTI的作 用可能发生在MAPK级联的上游 ${ }^{[55]}$ 。对这两种蛋白 结构的分析表明, AvrPto可能作为一个蛋白激酶抑 制剂起作用, 而AvrPtoB则是一个类泛素连接酶蛋 白。AvrPto能和FLS2, EFR及 BAK1 的激酶区域相互 作用, 这样的相互作用抑制了 PRRs的激酶活性 ${ }^{[56]}$, 并能干扰FLS2-BAK1 复合体形成 ${ }^{[57]}$ 。由于BAK1 可能 与多种PRRs相互作用, 阻止BAK1 与PRRs互作是效 应子抑制PTI的十分有效的策略。AvrPtoB泛素连接 酶活性与FLS2 泛素化及降解相关, 这是效应子在受 体水平抑制PTI的另一个策略 ${ }^{[58]}$ 。并不是所有细菌都 表达AvrPto 及 AvrPtoB，说明细菌还有其他抑制PTI 的策略。许多假单胞菌都产生HopAI1 蛋白, 它是一 个在动物及植物病原细菌中广泛存在且保守的效应 子 ${ }^{[59]}$ 。HopAI1 的表达使植物对P. syringae感病性增 加, 并能够抑制flg22 诱导的拟南芥基础抗性相关基 因 NHO1 的表达 ${ }^{[60]}$, 这些结果表明, HopAI1 是通过 靶定PAMPs介导的信号转导来抑制宿主防卫反应 的。后来研究证明, HopAI1 是一个磷酸苏氨酸裂解 酶, 能够将MAPK级联上的MPK3 和MPK6 去磷酸化, 从而终止PTI信号转导 ${ }^{[61]}$ 。

和细菌相比, 对于植物真核病原菌效应子的了 解较少。效应子既能在真菌和卵菌的细胞外基质中 作用, 也能在宿主细胞里作用。大部分已知的真菌 及卵菌效应子在其N末端都具有II型分泌信号肽，它 们能够由真核生物的分泌途径(II型分泌系统), 通过 胞吐作用分泌到细胞外基质 ${ }^{[62]}$ 。许多卵菌效应子在 
信号肽的下游有一个RXLR-(D)EER基序，它在胞吐 过程中不起作用, 但对效应子进入植物细胞是必需 的 ${ }^{[63,64]}$ 。然而，一些真菌效应子既没有RXLR区域， 也没有其他保守的多肽基序，它们同样能够进入植物 细胞 ${ }^{[65,66]}$, 其分子机制还有待于进一步研究。

\section{4 效应子的识别及 ETI}

病原微生物利用效应子攻克植物免疫系统的第 一道防线后, 在自然选择的压力下, 植物也进化出 了能够特异性识别这些效应子的受体, 开始启动另 一道免疫防线一一效应子触发的免疫(ETI)。R 基因 编码的产物 $\mathrm{R}$ 蛋白通常定位在植物细胞内, 这与它 们识别相应效应子的功能相符合。能够诱导 $\mathrm{R}$ 基因 抗性反应的这些病原物效应子基因被称为无毒 (Avirulence, Avr)基因。ETI 是基于 $\mathrm{R}$ 蛋白对 Avr 蛋 白直接或间接的识别而产生的, 因此也被称为基因 对基因的抗病性(Gene-for-gene resistance)。

大部分 $R$ 蛋白是具有多功能域的NB-LRR蛋白, 此类蛋白属于STAND超家族的一个亚家族。在动物 中, STAND超家族成员包括免疫、炎症及细胞调亡 的调控子 ${ }^{[67]}$ 。植物NB-LRR蛋白由一个多变的N末端, 一个核苷酸结合口袋(NB-ARC结构域)及C末端LRR 区域构成。根据NB-LRR蛋白N末端的不同, 又将其 分为两个亚类：一类是TIR-NB-LRR, 它们的N末端 与果蝇Toll及哺乳动物白细胞介素(IL)-1 受体的胞内 信号转导区域有同源性; 另一类是据推测能形成卷 曲螺旋(Coiled coil, CC)结构的CC-NB-LRR ${ }^{[68]}$ 。不同 的 $\mathrm{N}$ 末端需要不同的下游信号转导成分： TIR-NB-LRR下游是EDS1(Enhanced disease susceptibility 1), 而大部 分 CC-NB-LRR 则需要 NDR1(Non-race specific disease resistance 1)来激活 免疫应答 ${ }^{[69]}$ 。

对效应子的识别能够导致NB-LRR蛋白构象发 生改变, 将NB-LRR由抑制状态转变为激活状态, 从 而进一步激活下游信号转导 ${ }^{[70]}$ 。一般来讲, NB-LRR 采用间接及直接两种方式对效应子进行识别。一些 NB-LRR通过监督效应子对其靶标所产生的影响来 间接识别效应子。例如, 拟南芥的RIN4 是多种细菌 效应子(AvrRpt2、AvrRpm1、AvrB及HopF2)的靶标, 效应子致使RIN4 被修饰, 而至少有两种NB-LRR蛋 白((RPS2 和RPM1)能够识别RIN4 的改变, 进而触
发下游免疫应答。AvrRpt2 是一个能够直接裂解 RIN4 的蛋白酶, 它对 RIN4 的修饰作用激活了 NB-LRR蛋白RPS2 介导的ETI ${ }^{[71]}$; HopF2 是一个 ADP-核糖基转移酶, 它对RIN4 的修饰触发了植物 的ETI ${ }^{[72]}$; P. syringae中另外两个效应子AvrRpm1 和 AvrB 也能磷酸化 RIN4，而拟南芥 NB-LRR蛋白 RPM1 检测到RIN4 的磷酸化修饰, 触发ETI ${ }^{[73]}$ 。从 这些例子得知, 病原菌四个不相关效应子在植物中 的靶标都是RIN4, 暗示RIN4 对植物抗病具有重要 的作用。最近, 发现RIN4 能与 $\mathrm{H}^{+}$-ATP酶(AHA1 和 AHA2)共同调控气孔开闭, 从而影响细菌病原菌进 入叶片 ${ }^{[74]}$ 。AvrPhB是一个半胱氨酸蛋白酶, 它在植 物细胞内裂解丝氨酸-苏氨酸蛋白激酶PBS1, 从而 激活CC-NB-LRR蛋白PRS5, 触发ETI, 导致过敏性 细胞坏死反应 ${ }^{[75]}$ 。番茄的丝氨酸-苏氨酸蛋白激酶P to 是P. syringae AvrPto 和AvrPtoB的直接靶标, 在pto突 变体中, 这两个效应子都会增强病原菌致病性 ${ }^{[76]}$ 。Pto 要与NB-LRR蛋白Prf形成复合体, 才能激活最终产 生抗病反应的磷酸化级联信号 $[77]$ 。

与上述间接识别效应子的方式相对而言, NBLRR蛋白也能够与一些病原菌的效应子直接相互作 用。拟南芥RRS1-R蛋白以与茄科雷尔氏菌Ralstonia solanacearum效应子Pop2 直接相结合的方式来导致 ETI应答 ${ }^{[78]}$; 酵母双杂交实验证明亚麻的R蛋白L5、 L6 及L7 与亚麻锈病病菌Avr567 基因的产物具有相 互作用，体内实验也证明这种相互作用是特异的 ${ }^{[79]}$; 而亚麻锈病的另一个效应子 AvrM, 其C端结构域也 能直接与相应的NB-LRR蛋白 M相互作用 ${ }^{[80]}$; 稻瘟菌 Magnaporthe grisea 效应子 AvrPita直接与水稻 NB-LRR蛋白Pi-ta相结合并起始Pi-ta介导的防卫反 应 [81]。拟南芥 NB-LRR 蛋白 RPP1 和卵菌 Hyaloperonospora arabidopsidis效应子ATR1 的免疫 共沉淀实验证明, RPP1 LRR结构域与ATR1 的结合 对于ETI激活是必需的; 而在TIR或NB区域的突变不 能影响ATR1 结合, 却阻止了ETI应答 ${ }^{[82]}$, 这些结果说 明, 在直接识别过程中, LRR决定识别特异性, TIR和 NB结构域则负责激活下游信号转导。

当植物NB-LRR蛋白识别病原微生物效应子后, 是通过怎样的途径来激活下游免疫应答的呢? 目前 为止, 并没有一个清楚的模式来阐明这个信号转导 过程。近期一些研究表明, 细胞核内一些NB-LRR蛋 
白的激活对于触发防卫应答是必需的。大麦MLA10、 烟草N、拟南芥 RRS1-R、RPS4 及 SNC1 都需要在核 内定位与积累, 才能完全激活免疫应答 ${ }^{[69]}$ 。RRS1-R蛋 白上具有WRKY的 DNA结合区域, MLA10 蛋白能 与转录因子WRKY1 及WRKY2 相结合, 而 $\mathrm{N}$ 蛋白和 转录因子SPL相互作用 ${ }^{[83]}$ 。这些结果表明, NB-LRR 蛋白可能对免疫相关的转录调控有直接调节作用。

\section{5 结 语}

近年来，关于植物与病原微生物互作分子机制 的报道越来越多，人们对植物抗病机制的理解也更 加深入。和动物免疫系统相似, 植物免疫也是以 “自我”和“非我”的识别为基础，依靠 MAPK 级 联等信号网络, 将识别结果传递到细胞核内, 调控 相应基因表达，做出免疫应答。

病原微生物与植物共进化的结果, 使植物发展 出不同层次的免疫系统, 利用 PTI 及 ETI 抵抗不同 种类病原微生物的侵染。虽然 PTI 及 ETI 在植物先 天免疫过程中的重要作用已经被认知, 但还有许多 问题等待我们去研究及解答。参与 PTI 及 ETI 信号 转导过程中的许多信号分子及蛋白组份仍未知。植 物中存在许多的 RLKs，有多少是被用于感知 PAMPs 的 PRR? 一些 PRRs 和参与植物生长发育过 程的激素受体使用了相同的接头蛋白, 植物是怎样 保证下游信号转导的特异性 ? 一些潜在的 PRRs 属 于多基因家族，其中有很多紧密相关的家族成员， 它们之间的功能几余也造成了研究的困难。一些不 同的病原菌效应子能够靶定相同的宿主蛋白(如 RIN4)，这种情况在植物-病原微生物相互作用中是 普遍存在么? 植物中也存在着大量 NB-LRRs，它们 和不同病原菌效应子之间的对应关系又是如何呢? NB-LRRs 被激活的生化机制也不清楚。

开展植物与病原微生物间相互作用的研究, 不 仅可以加深我们对植物-病原微生物互作及交叉进 化等方面机制的认识, 而且可以为作物疾病防治及 抗病基因工程提供理论依据及策略, 具有十分重要 的意义。目前对植物 - 病原菌互作的认知主要来自 于对双子叶植物, 尤其是对模式植物拟南芥的研究, 而对于单子叶植物的抗病分子机理所知甚少; 许多 农作物都是单子叶植物, 因此对它们抗病机制的研 究急需进一步加强。此外, 随着高通量测序技术的
快速发展, 已经有很多学者着手于细菌、真菌及卵 菌的基因组研究, 这为进一步、广泛地研究植物与 病原微生物间的相互作用提供了便利条件。

\section{参考文献(References):}

[1] Jones JDG, Dangl JL. The plant immune system. Nature, 2006, 444(7117): 323-329. DOI

[2] Boller T, He SY. Innate immunity in plants: an arms race between pattern recognition receptors in plants and effectors in microbial pathogens. Science, 2009, 324(5928): 742-744. DOI

[3] Takken FLW, Tameling WIL. To nibble at plant resistance proteins. Science, 2009, 324(5928): 744-746. DOI

[4] Zipfel C. Pattern-recognition receptors in plant innate immunity. Curr Opin Immunol, 2008, 20(1): 10-16. DOI

[5] Naito K, Taquchi F, Suzuki T, Inagaki Y, Toyoda K, Shiraishi T, Ichinose Y. Amino acid sequence of bacterial microbe-associated molecular pattern flg22 is required for virulence. Mol Plant-Microbe Interact, 2008, 21(9): 1165-1174. DOI

[6] van de Veerdonk FL, Kullberg BJ, van der Meer JW, Gow NA, Netea MG. Host-microbe interactions: innate pattern recognition of fungal pathogens. Curr Opin Microbiol, 2008, 11(4): 305-312. DOI

[7] Postel S, Kemmerling B. Plant systems for recognition of pathogen-associated molecular patterns. Semin Cell Dev Biol, 2009, 20(9): 1025-1031. DOI

[8] Felix G, Duran JD, Volko S, Boller T. Plants have a sensitive perception system for the most conserved domain of bacterial flagellin. Plant J, 1999, 18(3): 265-276. DOI

[9] Chinchilla D, Bauer Z, Regenass M, Boller T, Felix G. The Arabidopsis receptor kinase FLS2 binds flg22 and determines the specificity of flagellin perception. Plant Cell, 2006, 18(2): 465-476. DOI

[10] Dunning FM, Sun WX, Jansen KL, Helft L, Bent AF. Identification and mutational analysis of Arabidopsis FLS2 leucine-rich repeat domain residues that contribute to flagellin perception. Plant Cell, 2007, 19(10): $3297-$ 3313. DOI

[11] Robatzek S, Bittel P, Chinchilla D, Köchner P, Felix G, Shiu SH, Boller T. Molecular identification and characterization of the tomato flagellin receptor LeFLS2, an orthologue of Arabidopsis FLS2 exhibiting characteristically different perception specificities. Plant Mol Biol, 2007, 64(5): 539-547. DOI

[12] Hann DR, Rathjen JP. Early events in the pathogenicity of Pseudomonas syringae on Nicotiana benthamiana. Plant $J$, 
2007, 49(4): 607-618. DOI

[13] Takai R, Isogai A, Takayama S, Che FS. Analysis of flagellin perception mediated by flg22 receptor OsFLS2 in rice. Mol Plant-Microbe Interact, 2008, 21(12): 1635-1642. DOI

[14] de Torres M, Mansfield JW, Grabov N, Brown IR, Ammouneh H, Tsiamis G, Forsyth A, Robatzek S, Grant M, Boch J. Pseudomonas syringae effector AvrPtoB suppresses basal defence in Arabidopsis. Plant J, 2006, 47(3): 368-382. DOI

[15] Zipfel C, Robatzek S, Navarro L, Oakeley EJ, Jones JD, Felix G, Boller T. Bacterial disease resistance in Arabidopsis through flagellin perception. Nature, 2004, 428(6984): 764-767. DOI

[16] Kunze G, Zipfel C, Robatzek S, Niehaus K, Boller T, Felix G. The $\mathrm{N}$ terminus of bacterial elongation factor $\mathrm{Tu}$ elicits innate immunity in Arabidopsis plants. Plant Cell, 2004, 16(12): 3496-3507. DOI

[17] Zipfel C, Kunze G, Chinchilla D, Caniard A, Jones JDG, Boller T, Felix G. Perception of the bacterial PAMP EF-Tu by the receptor EFR restricts Agrobacterium-mediated transformation. Cell, 2006, 125(4): 749-760. DOI

[18] Dallo SF, Kannan TR, Blaylock MW, Baseman JB. Elongation factor Tu and E1 $\beta$ subunit of pyruvate dehydrogenase complex act as fibronectin binding proteins in Mycoplasma pneumoniae. Mol Microbiol, 2002, 46(4): 1041-1051. DOI

[19] Granato D, Bergonzelli GE, Pridmore RD, Marvin L, Rouvet M, Corthésy-Theulaz IE. Cell surface-associated elongation factor Tu mediates the attachment of Lactobacillus johnsonii NCC533 (La1) to human intestinal cells and mucins. Infect Immun, 2004, 72(4): 2160-2169. DOI

[20] Lee Sw, Han SW, Sririyanum M, Park CJ, Seo YS, Ronald PC. A type I-secreted, sulfated peptide triggers XA21mediated innate immunity. Science, 2009, 326(5954): 850-853. DOI

[21] Ron M, Avni A. The receptor for the fungal elicitor ethylene-inducing xylanase is a member of a resistance- like gene family in tomato. Plant Cell, 2004, 16(6): 16041615. DOI

[22] Wang GD, Ellendorff U, Kemp B, Mansfield JW, Forsyth A, Mitchell K, Bastas K, Liu CM, Woods-Tör A, Zipfel C, de Wit PJGM, Jones JDG, Tör M, Thomma BPHJ. A genome-wide functional investigation into the roles of receptor-like proteins in Arabidopsis. Plant Physiol, 2008, 147(2): 503-517. DOI

[23] Ramonell K, Berrocal-Lobo M, Koh S, Wan JR, Edwards $\mathrm{H}$, Stacey G, Somerville S. Loss-of-function mutations in chitin responsive genes show increased susceptibility to the powdery mildew pathogen Erysiphe cichoracearum. Plant Physiol, 2005, 138(2): 1027-1036. DOI
[24] Fliegmann J, Mithöfer A, Wanner G, Ebel J. An ancient enzyme domain hidden in the putative $\beta$-glucan elicitor receptor of soybean may play an active part in the perception of pathogen-associated molecular patterns during broad host resistance. J Biol Chem, 2004, 279(2): 11321140. DOI

[25] Kaku H, Nishizawa Y, Ishii-Minami N, Akimoto-Tomiyama C, Dohmae N, Takio K, Minami E, Shibuya N. Plant cells recognize chitin fragments for defense signaling through a plasma membrane receptor. Proc Natl Acad Sci USA, 2006, 103(29): 11086-11091. DOI

[26] Miya A, Albert P, Shinya T, Desaki Y, Ichimura K, Shirasu K, Narusaka Y, Kawakami N, Kaku H, Shibuya N. CERK1, a LysM receptor kinase, is essential for chitin elicitor signaling in Arabidopsis. Proc Natl Acad Sci USA, 2007, 104(49): 19613-19618. DOI

[27] Wan JR, Zhang XC, Neece D, Ramonell KM, Clough S, Kim SY, Stacey MG, Stacey G. A LysM receptor-like kinase plays a critical role in chitin signaling and fungal resistance in Arabidopsis. Plant Cell, 2008, 20(2): 471481. DOI

[28] Gimenez-Ibanez S, Hann DR, Ntoukakis V, Petutschnig E, Lipka V, Rathjen JP. AvrPtoB targets the LysM receptor kinase CERK1 to promote bacterial virulence on plants. Curr Biol, 2009, 19(5): 423-429. DOI

[29] Hecht V, Vielle-Calzada JP, Hartog MV, Schmidt EDL, Boutilier K, Grossniklaus U, de Vries SC. The Arabidopsis SOMATIC EMBRYOGENESIS RECEPTOR KINASE 1 gene is expressed in developing ovules and embryos and enhances embryogenic competence in culture. Plant Physiol, 2001, 127(3): 803-816. DOI

[30] Wang WF, Kota U, He K, Blackburn K, Li J, Goshe MB, Huber SC, Clouse SD. Sequential transphosphorylation of the BRI1/BAK1 receptor kinase complex impacts early events in brassinosteroid signaling. Dev Cell, 2008, 15(2): 220-235. DOI

[31] Chinchilla D, Zipfel C, Robatzek S, Kemmerling B, Nürnberger T, Jones JDG, Felix G, Boller T. A flagellin-induced complex of the receptor FLS2 and BAK1 initiates plant defence. Nature, 2007, 448(7152): 497-500. DOI

[32] Heese A, Hann DR, Gimenez-Ibanez S, Jones AME, He K, Li J, Schroeder JI, Peck SC, Rathjen JP. The receptor-like kinase $\mathrm{SERK}_{3} / \mathrm{BAK}_{1}$ is a central regulator of innate immunity in plants. Proc Natl Acad Sci USA, 2007, 104(29): 12217-12222. DOI

[33] Akira S, Uematsu S, Takeuchi O. Pathogen recognition and innate immunity. Cell, 2006, 124(4): 783-801. DOI

[34] Veronese P, Nakagami H, Bluhm B, Abuqamar S, Chen X, Salmeron J, Dietrich RA, Hirt H, Mengiste T. The mem- 
brane-anchored BOTRYTIS-INDUCED KINASE1 plays distinct roles in Arabidopsis resistance to necrotrophic and biotrophic pathogens. Plant Cell, 2006, 18(1): 257-273. DOI

[35] Zhang J, Li W, Xiang TT, Liu ZX, Laluk K, Ding XJ, Zou Y, Gao MH, Zhang XJ, Chen S, Mengiste T, Zhang YL, Zhou JM. Receptor-like cytoplasmic kinases integrate signaling from multiple plant immune receptors and are targeted by a Pseudomonas syringae effector. Cell Host Microbe, 2010, 7(4): 290-301. DOI

[36] Lu DP, Wu SJ, Gao XQ, Zhang YL, Shan LB, He P. A receptor-like cytoplasmic kinase, BIK1, associates with a flagellin receptor complex to initiate plant innate immunity. Proc Natl Acad Sci USA, 2010, 107(1): 496-501. $\underline{\mathrm{DOI}}$

[37] MAPK Group, Ichimura K, Shinozaki K, Tena G, Sheen J, Henry Y, Champion A, Kreis M, Zhang SQ, Hirt H, Wilson C, Heberle-Bors E, Ellis BE, Morris PC, Innes RW, Ecker JR, Scheel D, Klessig DF, Machida Y, Mundy J, Ohashi Y, Walker JC. Mitogen-activated protein kinase cascades in plants: a new nomenclature. Trends Plant Sci, 2002, 7(7): 301-308. DOI

[38] Pitzschke A, Schikora A, Hirt H. MAPK cascade signalling networks in plant defence. Curr Opin Plant Biol, 2009, 12(4): 421-426. DOI

[39] Gao MH, Liu JM, Bi DL, Zhang ZB, Cheng F, Chen SF, Zhang YL. MEKK1, MKK1/MKK2 and MPK4 function together in a mitogen-activated protein kinase cascade to regulate innate immunity in plants. Cell Res, 2008, 18(12): 1190-1198. DOI

[40] Qiu JL, Zhou L, Yun BW, Nielsen HB, Fiil BK, Petersen K, MacKinlay J, Loake GJ, Mundy J, Morris PC. Arabidopsis mitogen-activated protein kinase kinases MKK1 and MKK2 have overlapping functions in defense signaling mediated by MEKK1, MPK4, and MKS1. Plant Physiol, 2008, 148(1): 212-222. DOI

[41] Andreasson E, Jenkins T, Brodersen P, Thorgrimsen S, Petersen NH, Zhu SJ, Qiu JL, Micheelsen P, Rocher A, Petersen M, Newman MA, Bjørn Nielsen H, Hirt H, Somssich I, Mattsson O, Mundy J. The MAP kinase substrate MKS1 is a regulator of plant defense responses. EMBO J, 2005, 24(14): 2579-2589. DOI

[42] Brodersen P, Petersen M, Bjørn Nielsen H, Zhu SJ, Newman MA, Shokat KM, Rietz S, Parker J, Mundy J. Arabidopsis MAP kinase 4 regulates salicylic acid- and jasmonic acid/ethylene-dependent responses via EDS1 and PAD4. Plant J, 2006, 47(4): 532-546. DOI

[43] Qiu JL, Fiil BK, Petersen K, Nielsen HB, Botanga CJ, Thorgrimsen S, Palma K, Suarez-Rodriguez MC, Sandbech-Clausen S, Lichota J, Brodersen P, Grasser KD,
Mattsson O, Glazebrook J, Mundy J, Petersen M. Arabidopsis MAP kinase 4 regulates gene expression through transcription factor release in the nucleus. EMBO J, 2008, 27(16): 2214-2221. DOI

[44] Ichimura K, Casais C, Peck SC, Shinozaki K, Shirasu K. MEKK1 is required for MPK4 activation and regulates tissue-specific and temperature-dependent cell death in Arabidopsis. J Biol Chem, 2006, 281(48): 36969-36976. $\underline{\mathrm{DOI}}$

[45] Asai T, Tena G, Plotnikova J, Willmann MR, Chiu WL, Gomez-Gomez L, Boller T, Ausubel FM, Sheen J. MAP kinase signalling cascade in Arabidopsis innate immunity. Nature, 2002, 415(6875): 977-983. DOI

[46] Pitzschke A, Schikora A, Hirt H. MAPK cascade signalling networks in plant defence. Curr Opin Plant Biol, 2009, 12(4): 421-426. DOI

[47] Ren DT, Liu YD, Yang KY, Han L, Mao GH, Glazebrook J, Zhang SQ. A fungal-responsive MAPK cascade regulates phytoalexin biosynthesis in Arabidopsis. Proc Natl Acad Sci USA, 2008, 105(14): 5638-5643. DOI

[48] Mao GH, Meng XZ, Liu YD, Zheng ZY, Chen ZX, Zhang SQ. Phosphorylation of a WRKY transcription factor by two pathogen-responsive MAPKs drives phytoalexin biosynthesis in Arabidopsis. Plant Cell, 2011, Epub ahead of print. DOI

[49] Citovsky V, Kapelnikov A, Oliel S, Zakai N, Rojas MR, Gilbertson RL, Tzfira T, Loyter A. Protein interactions involved in nuclear import of the Agrobacterium VirE2 protein in vivo and in vitro. $J$ Biol Chem, 2004, 279(28): 29528-29533. DOI

[50] Djamei A, Pitzschke A, Nakagami H, Rajh I, Hirt H. Trojan horse strategy in Agrobacterium transformation: abusing MAPK defense signaling. Science, 2007, 318(5849): 453-456. DOI

[51] Schulze-Lefert P, Panstruga R. Establishment of biotrophy by parasitic fungi and reprogramming of host cells for disease resistance. Annu Rev Phytopathol, 2003, 41: 641667. DOI

[52] Badel JL, Charkowski AO, Deng WL, Collmer A. A gene in the Pseudomonas syringae pv. tomato Hrp pathogenicity island conserved effector locus, hopPtoA1, contributes to efficient formation of bacterial colonies in planta and is duplicated elsewhere in the genome. Mol Plant-Microbe Interact, 2002, 15(10): 1014-1024. DOI

[53] Lindgren RB. The role of hrp genes during plant-bacterial interactions. Annu Rev Phytopathol, 1997, 35: 129-152. DOI

[54] Hauck P, Thilmony R, He SY. A Pseudomonas syringae type III effector suppresses cell wall-based extracellular defense in susceptible Arabidopsis plants. Proc Natl Acad 
Sci USA, 2003, 100(14): 8577-8582. DOI

[55] He P, Shan LB, Lin NC, Martin GB, Kemmerling B, Nürnberger T, Sheen J. Specific bacterial suppressors of MAMP signaling upstream of MAPKKK in Arabidopsis innate immunity. Cell, 2006, 125(3): 563-575. DOI

[56] Xiang TT, Zong N, Zou Y, Wu Y, Zhang J, Xing WM, Li Y, Tang XY, Zhu LH, Chai JJ, Zhou JM. Pseudomonas syringae effector AvrPto blocks innate immunity by targeting receptor kinases. Curr Biol, 2008, 18(1): 74-80. DOI

[57] Shan LB, He P, Li JM, Heese A, Peck SC, Nürnberger T, Martin GB, Sheen J. Bacterial effectors target the common signaling partner BAK1 to disrupt multiple MAMP receptor-signaling complexes and impede plant immunity. Cell Host Microbe, 2008, 4(1): 17-27. DOI

[58] Gohre V, Spallek T, Häweker H, Mersmann S, Mentzel T, Boller T, de Torres M, Mansfield JW, Robatzek S. Plant pattern-recognition receptor FLS2 is directed for degradation by the bacterial ubiquitin ligase AvrPtoB. Curr Biol, 2008, 18(23): 1824-1832. DOI

[59] Li XY, Lin HQ, Zhang WG, Zou Y, Zhang J, Tang XY, Zhou JM. Flagellin induces innate immunity in nonhost interactions that is suppressed by Pseudomonas syringae effectors. Proc Natl Acad Sci USA, 2005, 102(36): 1299012995. DOI

[60] Kang L, Li JX, Zhao TH, Xiao FM, Tang XY, Thilmony R, He Sy, Zhou JM. Interplay of the Arabidopsis nonhost resistance gene NHO1 with bacterial virulence. Proc Natl Acad Sci USA, 2003, 100(6): 3519-3524. DOI

[61] Zhang J, Shao F, Li Y, Cui HT, Chen LJ, Li HT, Zou Y, Long CZ, Lan LF, Chai JJ, Chen S, Tang XY, Zhou JM. A Pseudomonas syringae effector inactivates MAPKs to suppress PAMP-induced immunity in plants. Cell Host Microbe, 2007, 1(3): 175-185. DOI

[62] Panstruga R, Dodds PN. Terrific protein traffic: the mystery of effector protein delivery by filamentous plant pathogens. Science, 2009, 324(5928): 748-750. DOI

[63] Whisson SC, Boevink PC, Moleleki L, Avrova AO, Morales JG, Gilroy EM, Armstrong MR, Grouffaud S, van West P, Chapman S, Hein I, Toth IK, Pritchard L, Birch PRJ. A translocation signal for delivery of oomycete effector proteins into host plant cells. Nature, 2007, 450(7166): 115-118. DOI

[64] Dou DL, Kale SD, Wang X, Jiang RHY, Bruce NA, Arredondo FD, Zhang XM, Tyler BM. RXLR-mediated entry of Phytophthora sojae effector Avr1b into soybean cells does not require pathogen-encoded machinery. Plant Cell, 2008, 20(7): 1930-1947. DOI

[65] Catanzariti AM, Dodds PN, Lawrence GJ, Ayliffe MA, Ellis JG. Haustorially expressed secreted proteins from flax rust are highly enriched for avirulence elicitors. Plant
Cell, 2006, 18(1): 243-256. DOI

[66] Manning VA, Ciuffetti LM. Localization of Ptr ToxA produced by Pyrenophora tritici-repentis reveals protein import into wheat mesophyll cells. Plant Cell, 2005, 17(11): 3203-3212. DOI

[67] Lukasik E, Takken FL. STANDing strong, resistance proteins instigators of plant defence. Curr Opin Plant Biol, 2009, 12(4): 427-436. DOI

[68] Dangl JL, Jones JDG. Plant pathogens and integrated defence responses to infection. Nature, 2001, 411(6839): 826-833. DOI

[69] Elmore JM, Lin ZJD, Coaker G. Plant NB-LRR signaling: upstreams and downstreams. Curr Opin Plant Biol, 2011, 14(4): 365-371. DOI

[70] Collier SM, Moffett P. NB-LRRs work a "bait and switch" on pathogens. Trends Plant Sci, 2009, 14(10): 521-529. DOI

[71] Axtell MJ, Staskawicz BJ. Initiation of RPS2-specified disease resistance in Arabidopsis is coupled to the AvrRpt2directed elimination of RIN4. Cell, 2003, 112(3): 369-377. DOI

[72] Wilton M, Subramaniam R, Elmore J, Felsensteiner C, Coaker G, Desveaux D. The type III effector HopF2 Pto targets Arabidopsis RIN4 protein to promote Pseudomonas syringae virulence. Proc Natl Acad Sci USA, 2010, 107(5): 2349-2354. DOI

[73] Mackey D, Holt BF III, Wiig A, Dangl JL. RIN4 interacts with Pseudomonas syringae type III effector molecules and is required for RPM1-mediated resistance in Arabidopsis. Cell, 2002, 108(6): 743-754. DOI

[74] Liu J, Elmore JM, Fuglsang AT, Palmgren MG, Staskawicz BJ, Coaker G. RIN4 functions with plasma membrane $\mathrm{H}^{+}$-ATPases to regulate stomatal apertures during pathogen attack. PLoS Biol, 2009, 7(6): e1000139. DOI

[75] Shao F, Golstein C, Ade J, Stoutemyer M, Dixon JE, Innes RW. Cleavage of Arabidopsis PBS1 by a bacterial type III effector. Science, 2003, 301(5637): 1230-1233. DOI

[76] Abramovitch RB, Martin GB. AvrPtoB: a bacterial type III effector that both elicits and suppresses programmed cell death associated with plant immunity. FEMS Microbiol Lett, 2005, 245(1): 1-8. DOI

[77] Mucyn TS, Clemente A, Andriotis VME, Balmuth AL, Oldroyd GED, Staskawicz BJ, Rathjen JP. The tomato NBARC-LRR protein Prf interacts with Pto kinase in vivo to regulate specific plant immunity. Plant Cell, 2006, 18(10): 2792-2806. DOI

[78] Deslandes L, Olivier J, Peeters N, Feng DX, Khounlotham M, Boucher C, Somssich I, Genin S, Marco Y. Physical interaction between RRS1-R, a protein conferring resistance to bacterial wilt, and PopP2, a type III effector tar- 
geted to the plant nucleus. Proc Natl Acad Sci USA, 2003, 100(13): 8024-8029. DOI

[79] Dodds PN, Lawrence GJ, Catanzariti AM, Teh T, Wang CIA, Ayliffe MA, Kobe B, Ellis JG. Direct protein interaction underlies gene-for-gene specificity and coevolution of the flax resistance genes and flax rust avirulence genes. Proc Natl Acad Sci USA, 2006, 103(23): 8888-8893. DOI

[80] Catanzariti AM, Dodds PN, Ve T, Kobe B, Ellis JG, Staskawicz BJ. The AvrM effector from flax rust has a structured $\mathrm{C}$-terminal domain and interacts directly with the $\mathrm{M}$ resistance protein. Mol Plant-Microbe Interact, 2010, 23(1): 49-57. DOI

[81] Jia YL, McAdams SA, Bryan GT, Hershey HP Valent B.
Direct interaction of resistance gene and avirulence gene products confers rice blast resistance. EMBO J, 2000, 19(15): 4004-4014. DOI

[82] Krasileva KV, Dahlbeck D, Staskawicz BJ. Activation of an Arabidopsis resistance protein is specified by the in planta association of its leucine-rich repeat domain with the cognate oomycete effector. Plant Cell, 2010, 22(7): 2444-2458. DOI

[83] Caplan J, Padmanabhan M, Dinesh-Kumar SP. Plant NB-LRR immune receptors: from recognition to transcriptional reprogramming. Cell Host Microbe, 2008, 3(3): 126-135. DOI

\section{・综合信息・}

\section{“遗传学进步促进粮食安全与人口健康高峰论坛”通知}

中国的遗传学研究要面向国家的战略需求, 而粮食安全与人口健康是遗传学研究的重要领域, 为了交 流遗传学领域的新成果、新进展，中国遗传学会与七省二市遗传学会拟于 2012 年 8 月在河南省郑州市召开 “遗传学进步促进粮食安全与人口健康高峰论坛”。论坛将邀请我国遗传学及其相关研究领域的著名专家 和学者, 就近年来国内外遗传学最新研究进展和成果进行研讨和交流。会议将采取大会报告、分会报告和壁 报三种形式进行交流。欢迎从事遗传学及其相关学科研究的科研及教学工作者踊跃参加。

1. 主办单位: 中国遗传学会、河南省遗传学会、黑龙江省遗传学会、吉林省遗传学会、辽宁省遗传学 会、内蒙古遗传学会、北京市遗传学会、天津市遗传学会、河北省遗传学会、山西省遗传学会

2. 承办单位 : 河南农业大学、河南省遗传学会

3. 协办单位: 郑州大学、河南大学、河南师范大学、河南科技大学、河南科技学院、河南省农科院、 河南省人民医院

4. 征文内容: 医学遗传学、动物遗传学、植物遗传学、微生物遗传学、分子遗传学及基因组学等领域 的研究进展。论文摘要(500 1000 字)或全文, 格式参考《遗传》杂志稿约。优秀论文将推荐到《遗传》发表。 征文截止日期：2012 年 6 月 30 日。

5. 会议时间、地点

会议时间：2012 年 8 月中旬(具体日期见第二轮通知)

会议地点：河南省郑州市

6. 注册、提交论文摘要

提交征文之前先请注册报名, 网址：http://www.geneticssociety.cn,点击“遗传学进步促进粮食安全与人 口健康高峰论坛” 或直接登陆: http//ss.geneticssociety.cn进行注册报名。在论文提交登陆对话框中输入用户 名和密码后才能进行论文摘要的提交, 摘要格式请参考附件。

6. 注册、交通及食宿费

2012 年 6 月 30 日前注册报名的参会代表, 注册费为会员:800 元(凭会员证办入住手续); 其他代表 :900 元; 学生 700 元(凭学生证办入住手续)。现场注册各档次均增加 100 元。会议期间交通及食宿自理。

其他具体适宜详情见第二轮通知。 